\title{
Effects of Hearing Impairment on Listening Effort and Speech Intelligibility: A Systematic Review of Literature
}

\author{
Haroon Akram ${ }^{1} \quad$ Sohail Ahmed $^{1} \quad$ Xiaohui Yan $^{2} \quad$ Chenlu Wang $^{2} \quad$ Yonghua Wang $^{1} \quad$ Wendi Shi $^{2}$ \\ 1.Zhejiang Chinese Medical University, Hangzhou, 310053, Zhejiang Province, China \\ 2.Hangzhou Ren-ai Hearing Rehabilitation Research Centre, Hangzhou, China
}

\begin{abstract}
INTRODUCTION:

Hearing impairment is considered as the most common disorder in the humans as it causes lots of problems in routine life of people suffering from this ailment as it brings issues with speech recognition, communication, and language accretion Hearing impairment causes drastic effect on people's social life as it creates difficulties in and restricts them from participating in social activities due to increased effort required for listening
\end{abstract}

PURPOSE:

The main purpose of this study is to understand the effects of hearing impairment on listening effort and speech intelligibility

METHODS:

Literatures like Google Scholar, science Direct, PubMed were used and key words like cochlear implants, listening effort, speech intelligibility, and hearing loss, hearing impairment, Bilateral and unilateral hearing loss were used. Multiple retrospective, prospective and cross sectional studies were reviewed and additional methodological filters were used for paper selection and selected papers published from 2000 to 2021 were assessed and selected. 11 original articles fulfilled inclusion criteria

RESULTS:

After series of multiple experiments on different populations listening effort was found to be greater in subjects with hearing impairment, cochlear implant, Unilateral and bilateral hearing implants as compared to normal. Speech intelligibility is lower in subjects with high listening effort.

CONCLUSION:

Our review concluded that listening effort is inversely related to speech intelligibility. However no accurate technique is available to measure listening effort.

DOI: $10.7176 / \mathrm{JHMN} / 93-03$

Publication date:September $30^{\text {th }} 2021$

LIST OF ABBREVATIONS

\begin{tabular}{|c|c|}
\hline CI & Cochlear implant \\
\hline BI-CIs & Bilateral cochlear implants \\
\hline MBHL & Moderate bilateral hearing loss \\
\hline NR & Noise reduction \\
\hline NH & Normal hearing \\
\hline UHL & Unilateral hearing loss \\
\hline SNR & Signal to noise ratio \\
\hline VRT & Verbal response time \\
\hline
\end{tabular}

INTRODUCTION:

Hearing impairment is considered as the most common disorder in the humans as it causes multiple problems in routine life of people suffering from this ailment as it brings issues with speech recognition, communication, and language accretion this is due to degradation of internal acoustic stimuli. ${ }^{1}$ Many researchers stated in their studies that adverse effects of speech perception were higher in hearing impaired subjects as compared to normal-hearing listeners ${ }^{2,3}$

According to another estimation total number of school going children in USA is 5 million ${ }^{4} 12 \%$ of these children are suffering from some level of hearing loss that is likely to affect communication, learning, psychosocial development, and academic achievement $t^{5}$. Minimal hearing impairment is often associated with ear infections. However this minimum hearing impairment (MHI) was categorized in different groups as sensorineural know as most common cause of permanent or conductive or it can occur in only one ear called as unilateral. Also minimal sensorineural can also occur due to fluid in middle ear or wax build-up. ${ }^{6}$

Hearing impairment drastically affect people's social life as it creates difficulties in and restricts them from participating in social activities because increased effort is required for listening ${ }^{7-9}$. Subjective, behavioral and physiological methods are included to assess listening effort. Relation of listening effort with hearing 
impairment is considered an interesting topic by many researchers. ${ }^{10,11}$

\section{SEARCH STRATEGY:}

\section{METHODOLOGY}

Literatures like Google Scholar, science Direct, Pub Med were used and key words like cochlear implants, listening effort, speech intelligibility, and hearing loss, hearing impairment, Bilateral and unilateral hearing loss were used.

\section{STUDY SELECTION}

Multiple retrospective, prospective, cross sectional studies were reviewed and additional methodological filters were used for paper selection and selected papers published from 2000 to 2021 were assessed and selected. 11 original articles fulfilled inclusion criteria

\section{RESULT}

Total 11 original articles based on hearing impairment, listening effort and speech intelligibility+ are included in this review

Tina $\mathrm{M}$ et al conducted a study in 2008 on two groups of children ages ranging between 26 to 36 months. First group consists of 8 normal hearing children and second group of 18 children with sensorineural hearing loss. In second group of 10 deaf children bilateral and unilateral cochlear implant is used by 8 children. Single interval, minimum audible angles and 2-alternativeforced choice discrimination tasks were used to measure sensitivity. Observer-based psychophysical method is used to collect behavioral data. Primary results indicates that the observer-based psychophysical method is a useful method in normal hearing and deaf children with cochlear implants for measuring localization acuity and also emerging in toddlers with bilateral cochlear implants but not in toddlers with unilateral cochlear implants. ${ }^{12}$

A study conducted in 2007 involved 3 groups of cochlear implant and one normal hearing control group. The subjects underwent dual-task paradigm. First task was to identify sentences in noise and second task was to measure time of reaction on Stroop test. Speech recognition performance is $50 \%$ correct as performance was assessed for all subjects at signal to noise ratios (SNR) ranging 2-dB steps from 0 to $110 \mathrm{~dB}$ relative to an individual's SNR-50. Population was 46 adults who were sub divided in 4 groups, normal hearing group $(\mathrm{n}=12)$, unilateral cochlear implants $(\mathrm{n}=10)$, bi-lateral cochlear implants $(\mathrm{n}=12)$ and hybrid short electrode cochlear implants and bilateral residual hearing $(n=12)$. Remarkable differences were seen in speech intelligibility in the normal-hearing and the unilateral and bilateral cochlear implants groups. However there was no difference in earliest task performance among the hybrid cochlear implants and the control groups. Listening effort improved drastically in normal hearing group as compared to cochlear implants groups on all parts of six SNR conditions. However, there was no notable difference is seen in listening effort between the cochlear implant groups. The subjective measures revealed a notable difference in control and cochlear implants groups but no difference in between cochlear implant groups. Listening effort was not related with age at the onset of hearing loss, implantation, duration of implant being used and working memory capacity for this subjects. ${ }^{13}$

Study conducted by Pauquet et al used two groups unilateral mild to moderate hearing loss $(\mathrm{n}=20)$ and normal hearing age-matched participants $(n=19)$. A full-sentence working memory task manipulating demands on working memory and listening was studied. On the behavioral level, they discovered an extensive interplay of memory load and listening situation; this turned into, however, comparable for each groups. Under low, but not high memory load, listening condition significantly influenced task performance. Similarly, under easy but not difficult listening conditions, memory load had a significant effect on task performance. By using BOLD response it was found that increased response in the left supramarginal gyrus, left middle frontal gyrus, and left supplementary motor cortex is present under high memory load as compared to low memory load regardless of hearing ability. Increased responses found in the bilateral superior temporal gyrus under easy listening condition compared to difficult listening conditions. We found neither group differences nor interactions of group with memory load or with listening condition. This indicates that memory load and listening condition interact on a behavioral level, and only the increased memory load was seen in increased BOLD responses in frontal and parietal brain regions. So this study concluded that memory load should be considered while evaluating elderly patients as it may affect perfor mance. No further BOLD responses for age related hearing loss are found ${ }^{14}$

Anastoios et al conducted a study to test the hypothesis that positive effects of noise reduction might be to reduce cognitive effort directed toward speech reception, making it available for other tasks. 2 dual task experiments were conducted on normal hearing individuals. Fist task involves reporting words or sentences in noise set of different SNRs and second task involves memorizing words or responding in a complex visual reaction-time task. Noise reduction does not have any positive effect on speech intelligibility, but low values of signal-to-noise ratio, leads to better performance on second task. ${ }^{15}$

Lewis et al conducted a study in which two groups of children were involved. First group includes children 
of 5-12 years with normal hearing and second group of children 8-12 years of age with moderate bilateral hearing loss, unilateral hearing loss, or normal hearing. Consonant identification and word and sentence recognition performed in background noise. Percentage correct performance and verbal response time (VRT) were assessed. Speech recognition improves as SNR increases for all subjects. VRT measures were not different. Longer onset time is seen in incorrect response as compared to correct. In correct response VRT increases with decrease in SNR ${ }^{16}$.

Erin $\mathrm{M}$ conducted a study in 2017 on 16 adults with mild to severe hearing loss. Subjects were tested with study hearing aids of unidirectional, fixed directional and bilateral beam former settings. A dual-task paradigm used to assess objective listening effort and subjective ratings were used to assess subjective listening effort, in 2 signal-to-noise ratios. Low or moderate reverberation rooms were used while performing tests. Subjective and objective listening effort improve because of directional processing, moderate reverberation was beneficial in objective effort only. Word recognition performance was highly correlated with work and tiredness than objective listening effort. However, subjective ratings about control were significantly correlated with objective listening effort. ${ }^{17}$

Brons et al performed a study on 20 subjects with moderate sensorineural hearing loss. Subjects listened to speech in babble noise processed via noise reduction from three different linearly fitted hearing aids. Speechintelligibility tests, listening-effort ratings and paired ratings on noise annoyance, speech naturalness, and overall preference were performed on subjects. Noise reduction algorithms do not improved speech intelligibility, but reduced sound annoyance. The noise reduction that considered best with noise preference and annoyance had the worst intelligibility scores. The outcomes of intelligibility and listening comfort shows that preferred measurements might be useful as well in the selection of noise reduction. ${ }^{18}$

In Deniz et al research, (a) phonemic restoration as a measure of top-down filling of missing speech, (b) listening effort and response times as a measure of increased cognitive processing, and (c) visual world paradigm and eye gazing as a measure of the use of context and its time course were used to investigate cognitive compensation with hearing impairment, cochlear implants, and aging. Results of this research indicate that there is a fine balance between speech degradation and its cognitive compensation that vary greatly among individuals. Compensation benefits may limit due to inadequate hearing device settings. Cochlear implants seem to allow the effective use of sentential context, but likely at the cost of delayed processing. Verbal knowledge, which plays an important role in compensation, may be successfully employed in advanced age. These results shows that getting good compensation in hearing impairment is difficult and not impossible but also speech intelligibility tests aren't much reliable. ${ }^{19}$

Jonas et al conducted a study on 24 children with hearing impairment using cochlear implants and hearing aid all subjects were of age 6-13 years. A normal voice in quiet, a dysphonic voice in quiet, a typical voice in background noise and a dysphonic voice in background noise were the tests performed on all children. Voice quality and background noise increased listening effort in all subjects ${ }^{20}$.

Steel et al assessed binaural fusion on by conducting a study on 25 deaf children with cochlear implants and 24 with normal hearing. These subjects were asked if they heard one or two electric pulses $(250 \mathrm{~Hz}$ trains of 36 ms presented at $1 \mathrm{~Hz}$ ). Changes in pupil and reaction time were recorded to measure listening effort. Children with implants heard one image bilateral input less frequently than normal hearing subjects. Study outcomes indicate that without binaural level cues, children have difficulty compiling data from their bilateral implants to form one sound which causes increased listening effort. Brainstem asymmetries make this situation bad. Later implantation and longer access to bilateral acoustic hearing may support development of auditory pathways underlying binaural fusion. Improving bilateral cochlear implant signals for children is necessary to improve their binaural hearing. ${ }^{21}$

Adriana et al conducted a study on 38 adults with normal hearing. 3 SRT tests were performed in which they listened to sentences in stationary noise. One-up-one-down, two-up-one-down, or four-up one-down procedure was applied, resulting in the correct rehearsal of 50,71 , or $84 \%$ of the sentences. Peak dilation amplitude, the latency of the peak dilation amplitude, and the mean pupil dilation during the speech processing is measured in all conditions. Signal to noise ratio in SRT50\%, SRT71\%, and SRT $84 \%$ increased as a function of the speech intelligibility level. The subjective effort decreases, and the overall performance increases with level of speech intelligibility. In SRT50\% condition peak dilation amplitude and mean pupil dilation were higher as compared with the SRT71\% and SRT84\% conditions. The peak dilation amplitude, mean pupil dilation, and peak latency increased as SNR of speech decreases, but no effect on pupil diameter was observed. Response of pupil was higher for incorrect sentences than for correct sentences. The pupil response was greater in the first SRT test than in the $2^{\text {nd }}$ and $3^{\text {rd }}$ tests. Spearman correlation coefficients showed no link between the SNRs at the SRTs, subjective ratings, and the pupil response. ${ }^{22}$

\section{DISCUSSION:}

Keeping in view the results mentioned above hearing impairment is a common disorder that is often neglected. 
There is no objective method in clinical practice to assess listening effort. Early interventions can improve quality of life. Studies claim that the listening effort was lower in normal hearing groups in comparison to cochlear implant group weather implants were unilateral or bilateral. Also individuals with NH or MBHL or UHL have poor sentence recognition as SNR decreases. Another study states that some subjects claim subjective improvement from Noise reduction NR algorithms however no improvement in speech intelligibility is observed $^{15}$. It is concluded from another study that when peak dilation amplitude, peak latency, and mean pupil dilation increases, speech intelligibility decreases. However, it is necessary to find a standard measure to reduce listening effort in hearing impaired person as it benefits them and also further studies could be easier.

\section{CONCLUSION:}

After reviewing multiple studies we concluded that the speech intelligibility decreases with increase in listening effort. However many key points were missed out in this review, more study is needed to cover those points.

\section{REFERENCES}

1. Humes L. E \& Roberts L. (1990). Speech-recognition difficulties of the hearing impaired elderly: The contributions of audibility. J Speech Hear Res, 33, 726-735.

2. Hagerman, B. (1984). Clinical measurements of speech reception threshold in noise. Scand Audiol, 13, 5763.

3. Hopkins, K., Moore Brian, C. J., Stone Michael, A. (2005). Effects of Moderate Cochlear Hearing Loss on The Ability To Benefit From Temporal Fine Structure Information In Speech. J Acoust Soc Am, 123, $1140-1153$

4. National Association for the Education of Young Children. (2003). Critical facts about young children and early childhood programs in the United States. Rockville, MD: Author. Retrieved December 5, 2003, from http:// www.naeyc.org/childrens_champions/criticalbirth.asp

5. American Speech-Language-Hearing Association. (2003). The prevalence and incidence of hearing loss in children. Rockville, MD: Author. Retrieved December 5, 2003, from http://www.asha.org/public/hearing/disorders/ children.htm

6. Lynette R. Goldberg and Cynthia McCormick Richburg. Minimal Hearing Impairment: Major Myths With More Than Minimal Implications. Communication Disorders Quarterly 25:3 • pp. 152-160

7. Weinstein B. E, \& Ventry, I. M. (1982). Hearing impairment and social isolation in the elderly. J Speech Hear Res, 25, 593-599.

8. Demorest, M. E., \& Erdman, S. A. (1986). Scale composition and item analysis of the communication profile for the hearing impaired. J Speech Hear Res, 29, 515-535.

9. Strawbridge, W., Wallhagen, M. I., Shema, S. J., Kaplan, G. A. (2000). Negative consequences of hearing impairment in old age: A longitudinal analysis. Gerontologist, 40, 320-326.

10. Gosselin, P. A., \& Gagné, J. P. (2011). Older adults expend more listening effort than young adults recognizing speech in noise. J Speech Lang Hear Res, 54, 944-958

11. McGarrigle, R., Munro, K. J., Dawes, P., et al. (2014). Listening effort and fatigue: What exactly are we measuring? A British Society of Audiology Cognition in Hearing Special Interest Group 'white paper'. Int J Audiol, 53, 433-440

12. Tina M. Grieco-Calub, Ruth Y. Litovsky, Lynne A. Werner. Using the Observer-Based Psychophysical Procedure to Assess Localization Acuity in Toddlers Who Use Bilateral Cochlear Implants. Otology \& Neurotology 29:235Y239 2008, Otology \& Neurotology,

13. Ann E. Perreau Yu-Hsiang Wu Bailey Tatge et al. Listening Effort Measured in Adults with Normal Hearing and Cochlear Implants. J Am Acad Audiol 28:685-697 (2017)

14. Julia Pauquet, Christiane M. Thiel, Christian Mathys and Stephanie Rosemann. Relationship between Memory Load and Listening Demands in Age-Related Hearing Impairment. Hindawi Neural Plasticity Volume 2021, https://doi.org/10.1155/2021/8840452

15. Anastasios Sarampalis Sridhar Kalluri Brent Edwards, Ervin Hafter. Objective Measures of Listening Effort: Effects of Background Noise and Noise Reduction. Journal of Speech, Language, and Hearing Research Vol. 52 1230-1240 October 2009 D American Speech-Language-Hearing Association 10924388/09/5205-1230

16. Dawna Lewis, Kendra Schmid, Samantha O’Leary, Jody Spalding, Elizabeth Heinrichs-Graham, Robin High. Effects of Noise on Speech Recognition and Listening Effort in Children with Normal Hearing and Children With Mild Bilateral or Unilateral Hearing Loss. Journal of Speech, Language, and Hearing Research Vol. 59 1218-1232 October 2016

17. Erin M. Picou, Travis M. Moore, and Todd A. Ricketts. The Effects of Directional Processing on Objective and Subjective Listening Effort. Journal of Speech, Language, and Hearing Research Vol. 60 199-211 January 2017 
18. Inge Brons, Rolph Houben, and Wouter A. Dreschler. Effects of Noise Reduction on Speech Intelligibility, Perceived Listening Effort, and Personal Preference in Hearing-Impaired Listeners. DOI: $10.1177 / 2331216514553924$

19. Deniz Ba,skent, Jeanne Clarke, Carina Palsq et al. Cognitive Compensation of Speech Perception With Hearing Impairment, Cochlear Implants, and Aging: How and to What Degree Can It Be Achieved? DOI: $10.1177 / 2331216516670279$

20. K. Jonas Br€annstrom, Viveka Lyberg-Åhlandera, and Birgitta Sahl ena. Perceived listening effort in children with hearing loss: listening to a dysphonic voice in quiet and in noise. https://doi.org/10.1080/14015439.2020.1794030

21. Steel MM, Papsin BC, Gordon KA (2015) Binaural Fusion and Listening Effort in Children Who Use Bilateral Cochlear Implants: A Psychoacoustic and Pupillometric Study. PLoS ONE 10(2): e0117611. doi:10.1371/journal.pone.0117611

22. Adriana A. Zekveld, Sophia E. Kramer, and Joost M. Festen. Pupil Response as an Indication of Effortful Listening: The Influence of Sentence Intelligibility. Ear \& Hearing 2010;31;480- 490 